\title{
FLORA ARBUSTIVO-ARBÓREA DE UM FRAGMENTO DE MATA CILIAR NO ALTO RIO GRANDE, ITUTINGA, MINAS GERAIS ${ }^{1}$
}

\author{
Enivanis de Abreu Vilela ${ }^{2}$ \\ Ary Teixeira de Oliveira-Filho ${ }^{3}$ \\ Douglas Antônio de Carvalho ${ }^{2}$ \\ Manuel Losada Gavilanes ${ }^{2}$
}

Recebido em 01.09.92. Aceito em 06.02.95.

\begin{abstract}
RESUMO - (Flora arbustivo-arbórea de um fragmento de mata ciliar no alto rio Grande, Itutinga, Minas Gerais). Foi realizado um levantamento florístico numa área de $9.450 \mathrm{~m}^{2}$ de um fragmento de mata ciliar, margeando o reservatório da hidrelétrica de Camargos, no sul de Minas Gerais, Município de Itutinga. Foram levantados todos indivíduos que apresentavam diâmetros dos caules ao nível do solo de no mínimo $5 \mathrm{~cm}$ dentro da área amostral. Foram identificadas 253 espécies pertencentes a 66 famílias botânicas. São apresentados dados de similaridade desta mata com outras nos Estados de São Paulo e Minas Gerais.

Palavras-chave: Mata ciliar, levantamento florístico.

$$
\text { asis }
$$

ABSTRACT - (Woody flora of a fragment of riparian forest of the upper Rio Grande, Itutinga, state of Minas Gerais, Brazil) A floristic survey was carried out in an $9.450 \mathrm{~m}^{2}$ fragment of riparian forest on the margin of the hydroelectric reservoir of Camargos, Itutinga, state of Minas Gerais, S E Brazil. All individuals with minimum diameter at the base of stem $5 \mathrm{~cm}$ were surveyed in the sampled area forest. A list is provided with 253 species of trees and shrubs belonging to 66 botanic families. Floristic similarity indices were calculated in comparisons with other floristic surveys of forest in the states of São Paulo and Minas Gerais.
\end{abstract}

Key words: Riparian forest, floristic survey.

1 Projeto Integrado Mata Ciliar (Companhia Energética de Minas Gerais - CEMIG). CONTRATO: CEMIG/UFLA/FAEPE.

2 Professores do Departamento de Biologia - Universidade Federal de Lavras (UFLA) - Lavras - MG - CEP 37200-000.

3 Professor do Departamento de Ciências Florestais - Universidade Federal de Lavras (UFLA) - Lavras - MG - CEP 37200-000. 


\section{Introdução}

Nos últimos anos vêm ocorrendo uma maior conscientização no sentido de se protegerem as matas remanescentes, bem como de se revegetarem áreas degradadas no passado, com conseqüente aumento no esforço de pesquisa. Neste sentido, as matas ciliares vêm merecendo um destaque especial, não só pela sua importância para os cursos d'água, bem como pela influência que exercem sobre a fauna terrestre e aquática, além de seu efeito protetor das margens dos rios e reservatórios. Entretanto, as matas ciliares de Minas Gerais são pouco conhecidas, sendo que a maioria dos estudos existentes foi conduzida no Estado de São Paulo (Troppmair et al. 1970; Camargo et al. 1971; Camargo et al.1972; Troppmair \& Machado 1974; Gibbs \& Leitão Filho 1978; Gibbs et al. 1980; Bertoni et al. 1982; Bertoni \& Martins 1987). Também foram conduzidos naquele estado os primeiros trabalhos visando recuperar matas ciliares (Nogueira 1989, Barbosa et al. 1986, Salvador 1987, Joly 1989, Spigolon et al. 1989, Dematê 1989).

Em outros estados, como Mato Grosso, Ratter et al. $(1973,1978)$ descreveram as matas de galerias da Serra do Roncador e Oliveira-Filho \& Martins (1986) e Oliveira-Filho et al. (1990) levantaram a flora e a estrutura das matas de galeria da região da Chapada dos Guimarães, associadas a variáveis ambientais. No Distrito Federal, Ratter (1980) descreveu a vegetação de galeria da Fazenda Água Limpa, e Klein (1980), em Santa Catarina, caracterizou as matas ciliares do Vale do Itajaí. No Rio Grande do Sul, Bueno et al. (1987) estudaram a flora das margens do Rio Jacuí. No Paraná, foi realizado o inventário florestal do reservatório de Itaipu, sobre o qual foi elaborado o Projeto Gralha Azul (Klein et al., 1977), citados por Muller \& Zelazowski (1989), que consistiu no reflorestamento de suas margens.

Em Minas Gerais, estudos específicos e detalhados sobre matas ciliares estão sendo desenvolvidos muito recentemente, especialmente na região do Alto Rio Grande (Carvalho et al. 1992; Gavilanes et al. 1992). Devido à grande importância que o Rio Grande representa para o Estado de Minas Gerais, pelo seu potencial hidrelétrico e ao grande desmatamento ciliar nele ocorrido no passado, a Companhia Energética de Minas Gerais (CEMIG), Universidade Federal de Lavras (UFLA) e Fundação de Apoio ao Ensino, Pesquisa e Extensão (FAEPE), propuseram estudar as matas ciliares remanescentes na região do Alto Rio Grande, bem como outros tipos de cobertura florestal. Assim, este estudo é o terceiro de uma série de levantamentos florísticos realizados nesta região.

\section{Material e métodos}

A área estudada está situada no Município de Itutinga, sul de Minas Gerais, próxima às coordenadas $\left(21^{\circ} 21^{\prime} 50^{\prime \prime} \mathrm{S}\right.$ e $\left.44^{\circ} 37^{\prime} 00^{\prime \prime} \mathrm{W}\right)$, numa altitude de $917 \mathrm{~m}$ (Figura 1). O clima regional apresenta temperatura média anual de $19,4^{\circ} \mathrm{C}$, média de máximas de $28,2^{\circ} \mathrm{C}$ e o média de mínimas de $10,0^{\circ} \mathrm{C}$ (Vilela \& Ramalho, 1979). O clima segundo Koeppen é do tipo Cwb (mesotérmico, com inverno seco). 
A formação florestal estudada caracteriza-se, segundo Veloso et al. (1991), como Floresta Estacional Semidecídual Montana. Trata-se de um fragmento florestal com uma área total de 3,5 ha que é um prolongamento de antiga mata ciliar no Rio Grande, a qual foi submersa na sua parte mais baixa pelo reservatório da Hidrelétrica de Camargos, há mais de 30 anos. O solo sob a mata é um Cambissolo álico.

Foram amostrados $9.450 \mathrm{~m}^{2}$, distribuídos em 42 parcelas contíguas de $15 \times 15 \mathrm{~m}$, em dois blocos, sendo que um deles continha 20 parcelas e seu alinhamento seguia o declive natural da mata. O outro ligava-se ao primeiro por um ângulo de $90^{\circ}$. Todos indivíduos (árvores, arvoretas ou arbustos) que continham, ao nível do solo, diâmetro do caule $\geq 5 \mathrm{~cm}$, foram marcados e numerados com plaquetas de alumínio para posterior estudo fitossociológico. Além disso, em áreas adjacentes às parcelas, toda vez que se encontrava um espécime fértil, este era coletado. Após a coleta do material botânico, este foi levado para o herbário da UFLA para identificação, quando isto não era possível no campo. Os espécimes coletados foram prensados, secos, etiquetados, registrados e incorporados ao Herbário da Universidade Federal de Lavras (ESAL). Para identificação de algumas espécies, foram realizadas visitas aos herbários PAMG (Empresa de Pesquisa Agropecuária de Minas Gerais - EPAMIG, Belo Horizonte, MG); RB (Jardim Botânico do Rio de Janeiro, Rio de Janeiro, RJ) e UEC (Departamento de Botânica do Instituto de Biologia da Universidade Estadual de Campinas UNICAMP, Campinas, SP).

Foi calculada a similaridade florística com outras matas ciliares e semidecíduas dos Estados de São Paulo e Minas Gerais, através do índice de Jaccard (MuellerDombois \& Ellenberg, 1974).

\section{Resultados e discussão}

Foram identificadas 253 espécies, pertencentes a 66 famílias botânicas. As famílias que apresentaram maior número de espécies (superior a 7), por ordem decrescente, foram: Myrtaceae (30); Rubiaceae (15); Euphorbiaceae (14); Fabaceae e Lauraceae (12); Melastomataceae (10); Annonaceae, Asteraceae e Mimosaceae (8) (Tabela 1).

Comparando-se o levantamento florístico da mata de Itutinga com outros realizados nos Estados de São Paulo e Minas Gerais, observa-se que houve uma variação de 11,17 a 19,32\%. Apesar de os critérios adotados nos levantamentos florísticos de outros locais serem diferentes, os resultados obtidos neste estudo dão uma boa idéia da similaridade entre as diversas matas. Assim, entre as matas ciliares, a maior semelhança ocorreu com as de Ipeúna (19,32\%) e Mogi-Guaçu (16,32\%). No caso de matas semidecíduas, os maiores índices foram com Atibaia (16,98\%) e Guarulhos (16,81\%), induzido, talvez, em ambos os casos, pela maior proximidade com a região sul de Minas Gerais. Já o menor índice ocorreu com a mata de São José dos Campos, o que provavelmente se deve ao fato da maior proximidade desta mesma com a mata Atlântica sob características climáticas marcadamente diferentes (Tabela $2)$. 

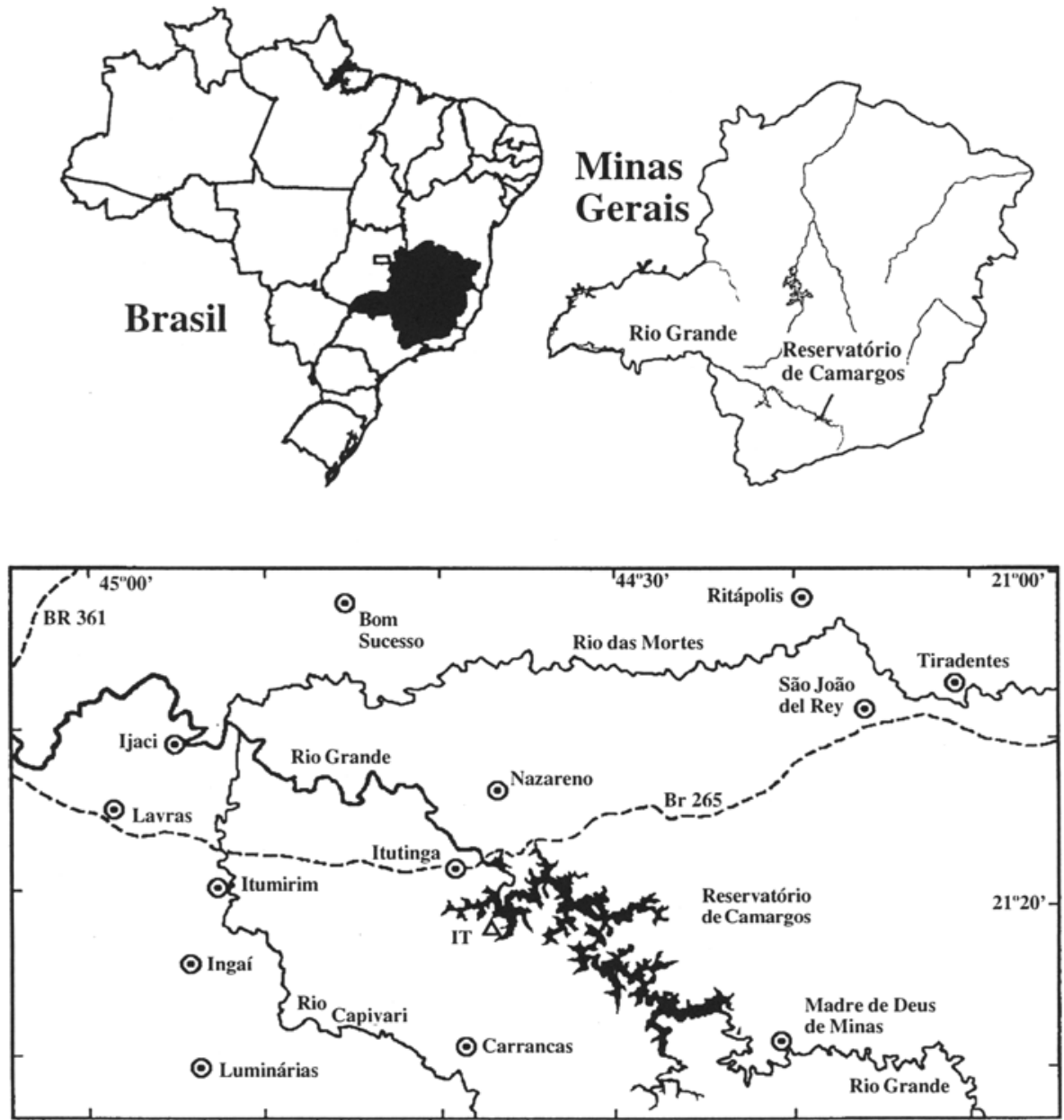

Figura 1. Situação geográfica da Mata de Itutinga (IT) às margens do Reservatório de Camargos, município de Itutinga, Estado de Minas Gerais. 
TABELA 1. Composição florística de mata semidecídua às margens do Reservatório de Camargos, Itutinga, MG. As espécies são listadas por família e em ordem alfabética e encontram-se acompanhadas de seu nome vernacular e do hábito com que foram encontradas com maior freqüência: A - árvore; a - arvoreta; b - arbusto.

\section{ANACARDIACEAE:}

Lithraea molleoides (Vell.) Engl.

aroeira-branca, aroeirinha

Schinus terebinthifolius Raddi

Tapirira guianensis Aubl.

Tapirira obtusa (Benth.) Mitchell

aroeira-fria, aroeira-vermelha a

fruta-de-pombo, pombeiro A

ANNONACEAE:

Annona cacans Warm.

Guatteria nigrescens Mart.

Rollinia emarginata Schlecht.

pombeiro-branco, pau-pombo

Rollinia mucosa (Jacq.) Baill.

Rollinia sericea R.E.Fries

Rollinia sylvatica Mart.

Xylopia brasiliensis Spreng.

Xylopia sericea St.Hil.

APOCYNACEAE:

Aspidosperma cylindrocarpon Muell. Arg.

Rauwolfia sellowii Muell. Arg.

AQUIFOLIACEAE:

Ilex conocarpa Reiss.

araticum-cagão

A

araticum-seco, pindaíba-preta a

araticunzinho A

araticum A

araticum-mirim, cortiça A

araticum-do-mato, cortiça A

pindaíba, pau-de-mastro A

pindarba, pimenteira A

peroba-poca A

casca-d'anta a

catuaba-do-mato, congonha A

ARACEAE:

Philodendron brasiliense Engl.

ARALIACEAE:

Dendropanax cuneatum (DC.) Decne \& Planch.

Schefflera calva (Cham.) D.Frodin

Schefflera macrocarpa (Cham.\& Schl.) D.Frodin

ARECACEAE:

Arecastrum romanzoffianum (Cham.) Becc.

Geonoma brevispatha Barb. Rodr.

Geonoma schottiana Mart.

Syagrus flexuosa (Mart.) Becc.

ASTERACEAE:

Baccharis dentata (Vell.) G.M.Barroso

Dasyphyllum brasiliense (Spr.) Mart.

Eremanthus incanus Less.

Gochnatia polymorpha (Less.) Cabrera

Piptocarpha axillaris (Less.) Baker

Piptocarpha macropoda Baker

Vanillosmopsis erythropappa Schultz

banana-do-brejo

b

mandioca, maria-mole A

mandiocão, morototó A

mandiocão $\quad \mathrm{A}$

jerivá, coco-baboso A

aricanga-do-brejo a

guaricanga, aricanga a

coquinho-baboso a

alecrim-de-árvore b

candeia-de-espinho a

candeinha, pau-de-candeia a

camará, candeia-podre a

vassoura-preta, canela-podre a

vassoura-preta a

candeia-verdadeira, cambará a 
Vernonia diffusa Less.

vassourão-preto a

Vernonia discolor Less.

BIGNONIACEAE:

Sparattosperma leucanthum K.Schum.

Tabebuia chrysotrycha (Mart.ex DC.) Standl.

Tabebuia impetiginosa (Mart.) Standl.

Tabebuia ochracea (Cham.) Rizz.

Tabebuia serratifolia (Vell.) Nichols

Tabebuia umbellata (Don.) Standel

BLECHNACEAE:

Blechnum brasiliense Desv.

BOMBACACEAE:

Ceiba speciosa (St.Hil.) Gibbs \& Semir

Eriotheca candolleana (K.Schum.) A.Robyns

Pseudobombax longiflorum (Mart. \& Zucc.) A. Robyns

Pseudobombax tomentosum (St.Hil.) A. Robyns

BORAGINACEAE:

Cordia rufescens A.DC.

Cordia sellowiana Cham.

Cordia superba Cham.

Cordia trichotoma (Vell.) Arrab. ex Steud.

\section{BURSERACEAE:}

Protium almecega March.

Protium heptaphyllum (Aubl.) March.

Protium widgrenii Engler

\section{CAESALPINIACEAE:}

Copaifera langsdorffii Desf.

Hymenaea courbaril L.

Peltophorum dubium (Spreng.) Taub.

Sclerolobium aureum (Tul.) Benth.

Senna multijuga (L.C.Rich.) Irwin \& Barneby

\section{CARICACEAE:}

Jacaratia spinosa (Aubl.) DC.

CECROPIACEAE:

Cecropia pachystachya Tréc.

\section{CELASTRACEAE:}

Austroplenckia populnea (Reiss.) Lund.

Maytenus aquifolium Mart.

Maytenus glazioviana Loes.

Maytenus gonoclados Mart.

Maytenus salicifolia Reiss.

CHLORANTHACEAE:

Hedyosmum brasiliense Mart.

vassourão-preto, cambará

ipê-cabeludo A

ipê-tabaco A

ipê-rosa, ipê-roxo A

ipê-cascudo, piúna-do-campo a

ipê-amarelo A

ipê-amarelo-do-brejo A

samambaia-do-brejo b

paineira A

pau-de-paina, catuaba-branca A

imbiruçu, paineira-lisa A

imbiruçu A

mulato-branco, bago-de-boi A

chá-de-bugre, juruté A

grão-de-galo a

canela-batata, louro-pardo A

almecega-de-casca-lisa, breu A

breu-vermelho A

almecega-cascuda A

copaiba-vermelha, pau-de-óleo A

jatobá-d'anta, jataí A

angico-cangalha A

pau-bosta, gonçalo-do-campo a

aleluia, canafístula A

jaracatiá A

embaúba-cinzenta A

carvalho-do-campo a

espinheira-santa a

coração-de-bugre, cafezinho A

congonha A

fruta-de-pomba, língua-de-tiú $\quad \mathrm{A}$

espirradeira-da-mata $\quad$ b 
CHRYSOBALANACEAE:

Hirtella hebeclada Moric ex A.DC.

araçá-da-serra, azeitona

CLETHRACEAE:

Clethra scabra Pers.

vermelhão, canjuja, vassourão

A

CLUSIACEAE:

Calophyllum brasiliense Camb.

Clusia criuva Camb.

Kielmeyera lathrophyton Saddi

Rheedia gardneriana Pl. \& Tr.

Vismia brasiliensis Choisy

COMBRETACEAE:

Terminalia glabrescens Mart.

CUNONIACEAE:

Lamanonia ternata Vell.

CYATHEACEAE:

Nephelea sternbergii (Sternb.) Tryon

Trichipteris phalerata (Mar.) Bar.

DICKSONIACEAE:

Dicksonia sellowiana (Presl.) Hook.

EBENACEAE:

Diospyros hispida A.DC.

guanandi, mangue A

estalo, criúva a

pau-santo a

bacupari-miúdo, bacoparé A

pau-de-lacre, purga-de-vento A

carvalho, mirindiba A

açoita-cavalo-vermelho A

samambaiaçu-branco b

samambaiaçu-vermelho b

xaxim b

marmelada, olho-de-boi A

ERYTHROXYLACEAE:

Erythroxylum campestre St.Hil.

Erythroxylum citrifolium St.Hil.

Erythroxylum cuneifolium (Mart.) Schulz.

EUPHORBIACEAE:

Acalypha brasiliensis Muell.Arg.

Actinostemon communis (Muell.Arg.) Pax

Alchornea glandulosa Poepp. \& Endl.

Aparisthmium cordatum (Juss.) Baill.

Croton echinocarpus Muell.Arg.

Croton floribundus Spreng.

Croton lobatus $\mathrm{L}$.

Croton urucurana Baill.

Hieronyma ferruginea Tul.

Pera obovata Baill.

Phyllanthus claussenii Muell.Arg.

Sapium longifolium Huber

Sebastiania brasiliensis Sprengel

Sebastiania edwalliana Pax \& Hoffmg.

Sebastiania serrata Muell.Arg.

fruta-de-tucano, garibaldi b

fruta-de-juriti $\quad b$

fruta-de-pomba b

acalifa b

laranjeira-brava a

tapiá, tanheiro A

pau-de-facho A

sangra d'água, sangue-de-drago A

tapichingui, capichingui A

mandioquinha a

sangra d'água, sangue-de-drago A

sangue-de-boi, quina-do-pará A

pau-de sapateiro, cacho-de-arroz A

quebra-pedra-grande $\quad \mathrm{b}$

visgueiro, leiteiro a

didale, visgueiro A

leiteiro a

esporão-de-espinho, branquinho A

FABACEAE:

Acosmium dasycarpum (Vog.) Yak.

sucupira-da-serra, chapada 
Acosmium sublegans (Vog.) Yak.

Andira anthelmia (Vell.) Macbr.

Bowdichia virgilioides H.B.K.

Dalbergia miscolobium Benth.

Machaerium aculeatum Raddi

Machaerium nictitans Benth.

Machaerium stipitatum (DC.) Vog.

Machaerium villosum Vog.

Ormosia fastigiata Tul.

Platycyamus regnellii Benth.

Platypodium elegans Vog.

FLACOURTIACEAE:

Casearia decandra Jacquin.

Casearia lasiophylla Eichl.

Casearia sylvestris Sw.

HIPPOCRATEACEAE:

Cheiloclinum cognatum (Miers.) A.C.Smith

LACISTEMACEAE:

Lacistema hasslerianum Chodat

LAMIACEAE:

Hyptis arborea Benth.

LAURACEAE:

Cryptocarya aschersoniana $\mathrm{Mez}$

Endlicheria paniculata (Spreng.) Macbr.

Nectandra cissiflora Meissn.

Nectandra grandiflora Nees

Nectandra nitidula Nees \& Mart. ex Nees

Nectandra oppositifolia Nees

Nectandra puberula (Schott.) Mez

Nectandra reticulata $\mathrm{Mez}$

Ocotea catharinensis $\mathrm{Mez}$

Ocotea corymbosa (Meissn.) Mez

Ocotea odorifera (Vell.) Rohwer

Ocotea pulchella (Nees) Mez

Persea pyrifolia Nees

LECYTHIDACEAE:

Cariniana estrellensis (Raddi) Kuntze

LOGANIACEAE:

Strychnos gardneri A.DC.

LYTHRACEAE:

Lafoensia pacari St.Hil.

MAGNOLIACEAE:

Talauma ovata St.Hil. sucupira-do-campo a

angelim-amargo, pau-de-morcego A

sucupira-preta A

cabiúna-do-campo a

adolfo, jacarandá-de-espinho A

jacarandá-ferro, bico-de-pato A

jacarandá-roxo, sapuva, ximbó A

jacarandá-mineiro $\mathrm{A}$

tento, mucunã A

pau-pereira, cataguá A

jacarandá-branco, faveiro A

canela-espeto, cambroé a

espeto-peludo a

erva-de-lagarto, guaçatonga a

bacupari a

cafeeiro-do-mato b

salva-do-campo b

canela-branca, canela-de-jacu A

canela-peluda, canela-do-brejo A

canela-amarela A

canela-sassafrás A

canela-amarela A

canela-amarela, canela-ferrugem A

louro-amarelo, fruta-de-jacu A

canela-amarela A

canelinha-preta A

canela-bosta, canela-preta A

canela-sassafrás, sassafrás A

canela-prego, canelinha A

massaranduba, nicurana A

jequitibá-branco, coatinga A

quina-de-cipó a

dedaleira a

pinha-do-brejo, baguaçu A 


\section{MALPIGHIACEAE:}

Heteropterys acutifolia A.Juss.

murici-de-rama

MALVACEAE:

Abutilon belfordianum (Hook.) St.Hil.\& Naud.

lanterninha

b

MELASTOMATACEAE:

Leandra scabra DC.

pixirica, camará-do-mato b

Miconia albicans $\mathrm{Tr}$.

canela-de-velho, olhos-de-porco b

Miconia corallina Spreng.

carvãozinho

Miconia cubatanensis Hoehne

carvãozinho

Miconia ligustroides (DC.) Naud.

jacatirão

A

Miconia minutiflora (Bonpl.) Tr.

pedra-uni, jacatirão a

Miconia pepericarpa DC.

Tibouchina candolleana (DC.) Cogn.

carvãozinho-vermelho a

quaresma a

Tibouchina stenocarpa (DC.) Cogn.

Trembleya parviflora (Don) Cogn.

MELIACEAE:

Cabralea canjerana (Vell) Mart.

Cedrela fissilis Vell.

Guarea guidonea (L.) Sleumer

Guarea kunthiana A.Juss.

Trichilia pallida Swartz.

MIMOSACEAE:

Albizia polycephala (Benth.) Killip

Anadenanthera colubrina (Vell.)Brenan

Anadenanthera peregrina (Benth.) Speg.

Inga affinis DC.

Inga luschnatiana Benth.

Inga marginata Willd.

Stryphnodendron polyphyllum Mart.

MONIMIACEAE:

Mollinedia argyrogyna Perk.

Mollinedia triflora (Spreng.) Tul.

Mollinedia uleana Perk.

Mollinedia widgrenii A.DC.

Siparuna apiosyce (Mart.) DC.

Siparuna guianensis Aubl.

MORACEAE:

Ficus gomelleira Kunth \& Bouché ex Kunth

Ficus insipida Willd.

Ficus luschnatiana (Miq.) Miq.

Maclura tinctoria (L.)Don. ex Stendel

Naucleopsis mello-barretoi (Standl.) C.C.Berg

quaresmão, cuiupeva a

flor-de-quaresma $\quad b$

cangerana, pindaiborana A

cedro-rosa, cedro-branco A

cura-madre, marinheiro a

guaré, ataúba A

catiguá $\mathrm{A}$

faveira, farinha-seca A

angico $\mathrm{A}$

angico-vermelho $\mathrm{A}$

ingá-doce, ingá-miúdo A

ingaçu $\mathrm{A}$

ingá-mirim a

barbatimão $\mathrm{A}$

congonha-branca a

capixim, pau-de-espeto b

cafeeiro-do-mato a

pimenteira-brava A

limoeiro-bravo, folha-santa b

negramina, capitiú a

gameleira A

figueira A

figueira A

amoreira, taiúva A

muiratinga A

canxim, cincho a 


\section{MYRSINACEAE:}

Myrsine coriacea (Sw.) R.Br.

Myrsine lancifolia Mart.

Myrsine umbellata Mart.

MYRTACEAE:

Blepharocalyx salicifolius (Kunth) Berg

Calyptranthes brasiliensis Spr.

Calyptranthes clusiaefolia (Miq.) Berg

Calyptranthes lucida Mart.ex DC.

Calyptranthes strigipes Berg

Campomanesia xanthocarpa Berg

Campomanesia guazumifolia (Camb.) Berg

Campomanesia pubescens (DC.) Berg

Campomanesia rufa (Berg.) Nied.

Eugenia florida DC.

Eugenia involucrata DC.

Eugenia pluriflora DC.

Eugenia punicifolia (Kunth) DC.

Gomidesia affinis (Camb.) Legr.

Gomidesia eriocalyx (DC.) Legr.

Gomidesia lindeniana Berg

Myrcia laruotteana Camb.

Myrcia leptoclada DC.

Myrcia multiflora (Lam.) DC.

Myrcia rostrata DC.

Myrcia rufipes DC.

Myrcia tomentosa (Aubl.) DC.

Myrcia velutina Berg

Myrcia venulosa DC.

Myrciaria tenella (DC.) Berg

Pimenta pseudocaryophyllus (Gomes) Landrum

Psidium cattleianum Sab.

Psidium guajava L.f.

Psidium guineense $\mathrm{Sw}$.

Siphoneugena widgreniana Berg

NYCTAGINACEAE:

Guapira noxia (Netto) Lundell

Guapira opposita (Vell.) Reitz.

PIPERACEAE:

Ottonia leptostachya Kunth

Piper aduncum L.

Piper arboreum Aubl.

Piper caracolanum C.DC. $\begin{array}{ll}\text { pororoca-branca } & \text { A } \\ \text { pororoquinha-branca } & \text { A } \\ \text { pororoca-branca } & \text { A }\end{array}$

guruçuca A

guamirim A

jaborandi A

jambo-do-mato, batinga-magra A

ruão a

guabiroba a

sete-capotes a

guabiroba-de-árvore a

guabiroba-de-árvore, casaca b

pimenteira A

pitanga-preta A

olho-de-boi a

murta-vermelha a

guamirim A

guamirim-orelhinha a

guamirim-de-folha-grande a

cocococa A

ingabaú A

cambuí A

guamirim-de-folha-miúda A

guamirim-lagoa a

goiabão, goiabeira-brava A

piúna, folha-miúda-cascuda A

guamirim-do-campo A

cambuí-preto b

craveiro-da-terra, cravo a

araçá-do-mato A

goiabeira a

araçá-preto, araçá-perinha a

cravinho, lagoa-miúda A

joão-mole, maria-mole a

maria-mole, flor-de-pérola A

jaborandi, jaguarundi b

erva de jaboti, aperta-mão b

fruto-de-morcego, caapeba b

fruto-de-morcego $\quad b$ 
Flora arbustiva-arbórea de um fragmento de mata ciliar

Piper cernuum A.DC.

Piper mollicomum Kunth.

PROTEACEAE:

Euplassa rufa (Loesen) Sleumer

Roupala brasiliensis Klotzsch

RHAMNACEAE:

Colubrina glandulosa Perkins

Rhamnus sphaerosperma Reiss.

ROSACEAE:

Prunus sellowii Koehne

RUBIACEAE:

Alibertia macrophylla Schum.

Amaioua guianensis Aubl.

Chomelia sericea Muell.Arg.

Coutarea hexandra (Jacq.) Schum.

Faramea cyanea Muell.Arg.

Guettarda uruguensis Cham. \& Schlecht

Guettarda viburnioides (Cham.) Schlecht

Ixora warmingii Muell. Arg.

Psychotria barbiflora DC.

Psychotria hastisepala Muell.Arg.

Psychotria sessilis (Vell.) Muell.Arg.

Psychotria suterella Muell.Arg.

Randia nitida (Kunth) DC.

Rudgea viburnioides (Cham.) Benth.

RUTACEAE:

Esenbeckia febrifuga (St.Hil.) A.Juss.

Galipea multiflora Schult.

Zanthoxylum rhoifolium Lam.

Zanthoxylum riedelianum Engl.

Zanthoxylum tingoassuiba Mart.

SAPINDACEAE:

Cupania racemosa (Vell.) Radlk.

Cupania vernalis Camb.

Diatenopteryx sorbifolia Radlk.

Matayba juglandifolia (Camb.) Radlk.

SAPOTACEAE:

Chrysophyllum marginatum (Hook \& Arn.) Radlk. SOLANACEAE:

Brunfelsia brasiliensis (Spreng.) Smith \& Downs

Cestrum laevigatum Schlecht

Solanum erianthum Don

Solanum granuloso-leprosum Dunal

caapeba-de-orelha
caapeba

b

b

catinga-de-barrão $\mathrm{A}$

carne-de-vaca, catucaém A

sobrasil, saguaraji-vermelho A

saguaraji-vermelho a

pessegueiro-bravo a

marmelada-de-cachorro A

marmelada, canela-de-veado A

veludo-branco b

veludo-preto b

cafezinho A

angélica, veludo-branco a

jangada, angélica A

ixora-do-mato A

erva-de-rato b

pau-de-espeto, araçá-bravo b

folha-miúda, azedeira, matadeira a

folha-miúda b

limão-bravo a

congonha-de-bugre b

mamoninha, guaxupita b

guamixinga, grumarim a

mamica-de-porca A

maminha-de-porca A

maminha-de-porca A

caguatã, camboatã, pavão A

pau-de-cantil, gragoatã A

maria-preta, cansa-crioulo A

caqui-do-mato, cragoatã-branco A

gumbijava, guatambu de leite a

manacá b

dama-da-noite, coeirana b

jurubeba-de-árvore a

jurubeba-de-árvore a 
Solanum martii Sendt.

STERCULIACEAE:

Helicteres ovata Lam.

Guazuma ulmifolia L.

STYRACACEAE:

Styrax camporum Pohl

SYMPLOCACEAE:

Symplocos pubescens Klotzsch

THEACEAE:

Laplacea semiserrata Camb.

THYMELAEACEAE:

Daphnopsis brasiliensis Mart. \& Zucc.

Daphnopsis fasciculata (Meissn.) Nevl.

TILIACEAE:

Luehea grandiflora Mart. \& Zucc.

Luehea paniculata Mart.

Luehea rufescens St.Hil.

VERBENACEAE:

Aegiphilla lhotzkiana Cham.

Aegiphilla sellowiana Cham.

Cytharexylum myrianthum Cham.

Lantana fucata Lindl.

Vitex polygama Cham.

VOCHYSIACEAE:

Qualea multiflora Mart.

Vochysia tucanorum Mart. braço-de-mono, marianeira

b

saca-rolha, guaxima

a

pau-de-motamba, camacã

A

sabão, benjoim, canelão

A

sete-sangrias, saboeiro

A

mangue

A

imbira-branca, imbira-de-sapo

A

imbira-vermelha

A

açoita-cavalo

A

açoita-cavalo

a

açoita-cavalo

A

pau-de-papagaio

b

briaúva, pau-de-tamanco a

cinzeiro-preto a

alecrim-de-árvore

b

maria-preta, velame-do-campo

A

bagre, pau-terra, cinzeiro

A

pau-de-tucano, congonha-murici

TABELA 2 - Número de espécies arbóreas (NA) encontradas em levantamentos florísticos de matas dos estados de São Paulo e Minas Gerais, número de espécies em comum (NC) com o presente levantamento e índices de similaridade de Jaccard (IJ).

\begin{tabular}{llllll}
\hline LOCAL & AUTOR(ES) & TIPO DE MATA & NA & NC & IJ(\%) \\
\hline Ipeúna/SP & Rodrigues, 1992 & Ciliar & 204 & 74 & 19,32 \\
Brotas/SP & Salis, 1990 & Ciliar & 82 & 39 & 13,18 \\
Mogi-Guaçu/SP & Mantovani et al., 1980 & Ciliar & 139 & 55 & 16,32 \\
Uberlândia/MG & Schiavini, 1992 & Ciliar & 100 & 44 & 14,24 \\
Atibaia/SP & Meira Neto et al., 1989 & Semidecídua & 181 & 63 & 16,98 \\
Jundiaí/SP & Rodrigues, 1986 & Semidecídua & 206 & 60 & 15,04 \\
Guarulhos/SP & Gandolfi, 1991 & Semidecídua & 150 & 58 & 16,81 \\
S.J.dos Campos/SP & Silva, 1989 & Semidecídua & 195 & 45 & 11,17 \\
\hline
\end{tabular}




\section{Referências bibliográficas}

Barbosa, L.M., Barbosa, J.M., Batista, E.A., Mantovani, W., Veronese, S.A. \& Andreani Jr, R. 1989. Ensaios para estabelecimentos de modelos para recuperação de áreas degradadas de matas ciliares, Mogi-Guaçu(SP) - Nota prévia In: Simpósio Sobre Mata Ciliar, São Paulo, SP, 1989. Anais... p.268283.

Bertoni, J.E.A. \& Martins, F.R. 1987. Composição florística de uma floresta ripária na Reserva Estadual de Porto Ferreira, SP. Acta bot. bras., 1:17-16.

Bertoni, J.E.E., Stublebine, W.H., Martins, F.R. \& Leitão Filho, H.F. 1982. Nota prévia: Comparação fitossociológica das principais espécies de florestas de terra firme e da várzea na Reserva Estadual de Porto Ferreira (SP). In: Congresso Nacional Sobre Essências Nativas, Campos de Jordão, SP, set. 12.18, 1982. Anais... Silvicultura em São Paulo, 16A:563-571 (Edição Especial).

Bueno, O.L., Neves, M.T.M.B., Oliveira,M.L.A.A., Ramos, R.L. \& Strehl,T. 1987. Florística em áreas da margem direita do Baixo Jacuí, RS, Brasil, Acta bot. bras., 1:101-121.

Camargo, J.C.G., César, A.L., Gentil, J.P., Pinto, S.A.F \& Troppmair, H. 1971. Estudo fitogeográfico de vegetação ciliar do Rio Corumbataí (SP). Série biogeográfica n- 3. Instituto de Geografia, USP, São Paulo.

Camargo, J.C.G., Pinto, S.A.F. \& Troppmair, H. 1972. Estudo fitogeográfico e ecológico da bacia hidrográfica paulista do Rio da Ribeira. Instituto de Geografia, USP, Série Biogeografia 5, São Paulo.

Carvalho, D.A., Oliveira-Filho, A.T., Vilela, E.A. \& Gavilanes, M.L. 1992. Flora arbustivo-arbórea das matas ciliares do Alto Rio Grande o (MG). 1 - Mata de Macaia (Bom Sucesso). In: 2- Congresso Nacional Sobre Essências Nativas, São Paulo, SP. 1992. Anais... p.274-281.

Dematê, M.E.S.P. 1989. Recomposição de matas ciliares na região de Jaboticabal, SP. In: Simpósio Sobre Mata Ciliar, São Paulo, SP. 1989. Anais...p.160-170.

Gandolfi, S. 1991. Estudo florístico e fitossociológico de uma floresta residual na área do Aeroporto Internacional de São Paulo, Guarulhos, SP. Dissertação de Mestrado, UNICAMP, Campinas-SP.

Gavilanes, M.L., Oliveira-Filho, A.T., Carvalho, D.A. \& Vilela, E.A. 1992. Flora arbustivo-arbórea de uma mata ciliar do Alto Rio Grande, em Madre de Deus de Minas-MG. Daphne, 2(4):15-24.

Gibbs, P.E. \& Leitão Filho, H.F. 1978. Floristic composition of an area of gallery forest near Mogi-Guaçu, state of São Paulo, S.E. Brazil. Revta bras. bot., 1(1):151-156.

Gibbs, P.E., Leitão Filho, H.F. \& Abbott, R.I. 1980. Aplication of the paint-centred quarter method in a floristic survey of an area of gallery forest at Mogi-Guaçu, SP, Brazil. Revta bras. bot., 3(1/2):17-22.

Joly, C.A. 1989. Recomposição das matas ciliares do Rio Jacaré-Pepira: uma experiência prática. XI Congresso Nacional de Botânica. Resumo.p.638. Cuiabá-MT.

Klein, R.M. 1980. Ecologia da flora e vegetação do vale do Itajaí. Sellowia: v32, n32. Itajaí-SC.

Mantovani W., Rossi, L., Romaniuc Neto, S., Assad-Ludewigs, I.Y., Wanderlei, M.G.L., Meld, M.M.R.F. \& Toledo, C.B. 1989. Estudo fitossociológico de áreas de mata ciliar em Mogi-Guaçu, SP, Brasil. In: Barbosa, L.M. (Coord.). Simpósio Sobre Mata Ciliar, Anais. Campinas, Fundação Cargill, p.235283.

Meira Neto, J.A.A., Bermacci, L.C., Grombone, M.T., Tamashiro, L.Y. \& Leitão Filho, H.F. 1989. Composição florística da floresta semidecídua de altitude do Parque Municipal da Grota Funda (Atibaia, Estado de São Paulo). Acta bot. bras., 3(2):51-74.

Mueller-Dombois, D \& Ellenberg, H. 1974. Aims and methods of vegetation ecology. New York, John Wiley \& Sons.

Muller, A.C. \& Zelazowski, V.H. 1989. Reflorestamento Ecológico da faixa de proteção do Reservatório de Itaipú - ME. In: Simpósio Sobre Mata Ciliar, São Paulo, SP, 1989. Anais... p.213-232.

Nogueira, J.C.B. 1989. Recomposição da mata ciliar da Usina Estér em Cosmópolis, reserva florestal de Bauru (Instituto Florestal) e Oeste do Estado de São Paulo. In: Simpósio Sobre Mata Ciliar, São Paulo, SP. 1989. Anais... p.156-159. 
Oliveira-Filho, A.T. \& Martins, F.R. 1986. Distribuição, caracterização e composição das formações vegetais da região da Salgadeira, na Chapada dos Guimarães (MT). Revta bras. bot., 9:207-223.

Oliveira-Filho, A.T., Ratter, J.A. \& Shepherd, G.J. 1990. Floristic composition and community structure of a Central Brazilian gallery forest. Flora 184:103-117.

Ratter, J.A. 1980. Notes on the vegetation of Fazenda Água Limpa (Brasilia, DF. Brazil). Edinburg, Royal Botanic Gardin.

Ratter, J.A., Richards, P.W., Argent, G. \& Gifford, D.R. 1973. Observations on the vegetation of northeastern Mato Grosso - Expedition area. Philosophical Transactions of the Royal Society of London, B. Biological Sciences, 266:449-492.

Ratter, J.A., Askew, G.P., Montegomery, R.F. \& Gifford, D.R. 1978. Observations on forests of some mesotrophic soils in Central Brazil. Revta bras. bot., 1:47-58.

Rodrigues, R.R. 1986. Levantamento florístico e fitossociológico das matas da Serra do Japi, Jundiaí, $S P$. Dissertação de Mestrado. Campinas, Universidade Estadual de Campinas.

Rodrigues, R.R. 1992. Análise de um remanescente de vegetação natural às margens do Rio Passa Cinco, Ipeúna, SP. Tese de Doutorado, UNICAMP, Campinas, SP.

Salis, S.A. 1990. Composição florística e estrutura de um remanescente de mata ciliar do Rio JacaréPepira, Brotas, SP. Dissertação de Mestrado, UNICAMP, Campinas, SP. $111 \mathrm{p}$.

Salvador, J.L.G. 1987. Considerações sobre as matas ciliares e a implantação de reflorestamento mistos nas margens de rios e o reservatórios. Série Divulgação e Informação n. ${ }^{\circ}$ 5. Companhia Energética de São Paulo - CESP.

Silva, A.F. 1989. Composição florística e estrutura fitossociológica do estrato arbóreo da Reserva Florestal Professor Augusto Ruschi, São José dos Campos, SP. Tese de Doutorado. Universidade Estadual de Campinas. Campinas.

Schiavini, I.S. 1992. Estrutura das comunidades arbóreas de mata de galeria da Estação Ecológica do Panga-Uberlândia, MG. Tese de Doutorado, UNICAMP, Campinas.

Spigolon, J.R., Lieberg, S.A. \& Joly, C.A. 1989. Recuperação das matas ciliares da bacia do Rio JacaréPepira, Estado de São Paulo. XL Congresso Nacional de Botânica. Resumo. p. 522. Cuiabá - MT.

Troppmair, H., Camargo, J.C.G. \& Ferreira Pinto, S.A. 1970. Contribuição ao estudo fitogeográfico e ecológico da vegetação ciliar do alto e médio Corumbataí, São Paulo, Caderno de Ciências da Terra. IG/USP, 5:19-24.

Troppmair, H. \& Machado, M.L.A. 1974. Variação da estrutura da mata-galeria na bacia do Rio Corumbataí (SP), em relação à água no solo, de tipo de margem e do traçado de rio. Série Biogeografia n. ${ }^{\circ}$ 8. Instituto de Geografia, USP, São Paulo.

Veloso, H.P., Rangel Filho, A.L.R., Lima, J.C.A. 1991. Classificação da vegetação brasileira, adaptada a um sistema universal. IBGE.

Vilela, E.A. \& Ramalho, M.A.P. 1979. Análise das temperaturas e precipitações pluviométricas de Lavras, Minas Gerais. Ciência e Prática, 3:71-79. 